\title{
Evaluation Regarding Sites and Types of Adhesion in Previous Cesarean Sections: A study in a Tertiary Care Hospital
}

\author{
Dr. Sabina Parveen ${ }^{1 *}$, Dr. Md. Mahmudur Rahman², Dr. Begum Shamsun Naher Sherin ${ }^{3}$, Dr. Mahbuba Akhter ${ }^{4}$
}

\author{
${ }^{1}$ Assistant Professor, Department of Gynaeocology \& Obstetrics, US-Bangla Medical College \& Hospital, Bangladesh \\ ${ }^{2}$ Deputy Director (CS) \& PM(SD), CCSD, Directorate General of Family planning, Dhaka, Bangladesh \\ ${ }^{3}$ Associate Professor, Department of Gynaeocology \& Obstetrics, US-Bangla Medical College \& Hospital, Bangladesh \\ ${ }^{4}$ Professor, Department of Gynaeocology \& Obstetrics, US-Bangla Medical College \& Hospital, Bangladesh
}

DOI: $10.36347 /$ sjams.2020.v08i09.038

| Received: 17.09.2020 | Accepted: 25.09.2020 | Published: 28.09.2020

*Corresponding author: Dr. Sabina Parveen

Abstract

Original Research Article

Introduction: During present decades caesarean section surgery has become one of the most common obstetric operations worldwide. Adhesions developed after cesarean sections (CS) vary according to the number of repeat section. We have very few data regarding adhesion locally. Aim of the study: The aim of this study was to evaluate the adhesion in previous cesarean sections regarding the sites and types of adhesion. Methods: This is a prospective observatory study conducted in the department of Obstetrics and Gynaecology in US-Bangla Medical College Hospital, Dhaka, Bangladesh during the period from January 2019 to December 2019. The study was approved by the ethical committee of the mention hospital. The proper written consents were obtained from all the participants before starting the main intervention. In total 60 patients with adhesion in previous cesarean sections were selected as the total study population of this study. Results: The most common type of adhesions was uterovesical found in all cases $(100 \%)$. Omental adhesions with the anterior uterine wall came next in 56 cases $(93.33 \%)$. Case of uterosigmoid was $18.33 \%$ and the case with the uterus intestinal was $11.67 \%$ in this study. Besides all these findings in analyzing the sites of adhesions we found the highest $70 \%$ cases of avascular. Then both fine and thin were $38.33 \%$ separately. On the other hand the frequencies of dense, thick, and vascular and distort anatomy were $58.33 \%, 45 \%, 28.33 \%$ and $21.67 \%$ respectively. Conclusion: A high percentage of cesarean deliveries result in adhesive disease, which delays repeat cesarean delivery of the fetus. The potential for adhesive disease should be included in counseling regarding primary elective cesarean births. The findings may be helpful in the treatment arena and in farther studies.

Keywords: Adhesion, Cesarean Sections, Uterus Sheaths, Maternal morbidity.

Copyright @ 2020: This is an open-access article distributed under the terms of the Creative Commons Attribution license which permits unrestricted use, distribution, and reproduction in any medium for non-commercial use (NonCommercial, or CC-BY-NC) provided the original author and source are credited.

\section{INTRODUCTION}

A high percentage of cesarean deliveries result in adhesive disease, which delays repeat cesarean delivery of the fetus. The potential for adhesive disease should be included in counseling regarding primary elective cesarean births. The findings may be helpful in the treatment arena and in farther studies. Adhesions are defined as an abnormal fibrous connection between 2 anatomically different surfaces area [1]. They often occur as a result of surgery and formed between internal organs and tissues, joining them together. Postoperative adhesions frequently occur following abdominal surgery and are linked with a large economic burden due to economic costs of the length of hospital stay resulting from adhesiolysis [2]. Now a day, adhesiolysis because of cesarean birth become very common and primary elective cesarean births may be on the rise, with considerable national debate focused on the role of cesarean deliveries in the prevention of pelvic relaxation and the potential negative impact of vaginal deliveries on long-term maternal health [3-5]. Adhesions after CS or any laparotomy is associated with increased rates of chronic pelvic pain, infertility, bowel obstruction and ectopic pregnancy. Pelvic adhesion can influence the maternal morbidity which can occur in women with repeat CS, and can change the decision from total hysterectomy to subtotal hysterectomy. Also, little can influence the incidence of bowel and bladder injury during hysterectomy [6]. Adhesion during current CS is of great value in preventing adhesions in next CS especially with the unavailability of anti-adhesion barriers which may be used extensively in developed countries to prevent future adhesion [7]. Adhesion can be done by scissors dissection and ligation of fibrous bands or using monopolar diathermy coagulation. Electrocoagulation produces severe trauma with subsequent acute and 
prolonged inflammatory reactions [8] increasing postsurgical adhesion formation. The aim of this study was to evaluate the adhesion in previous cesarean sections regarding the sites and types of adhesion. All the parts of this study were performed according to the objectives of this study.

\section{OBJECTIVES}

\section{a) General objective}

- To evaluate the adhesion in previous cesarean sections regarding the sites and types of adhesion.

\section{b) Specific Objectives}

- To know more about the present conditions of adhesion in previous cesarean sections in Bangladesh.

\section{Methodology \& Materials}

This was a prospective observatory study which was conducted in the Department of Obstetrics and Gynaecology in US-Bangla Medical College Hospital, Dhaka, Bangladesh during the period from January 2019 to December 2019. All eligible participants signed a written informed consent before participation after explaining the purpose of the study. In total 60 pregnant women at term (37-40 weeks) gestation with uncomplicated singleton pregnancy scheduled for elective repeated lower segment cesarean section under spinal anesthesia were included in the study. Pregnant women with medical disorders, placenta previa, any contraindication to spinal anesthesia, and those who refused to participate in the study were excluded. Patients' age, parity, gestational age and the number of previous CS were registered before the start of surgery. All cesarean deliveries were conducted by a staff member (the two authors) with an assistant senior resident. Starting CS after good disinfection of the skin from xiphisternum to the suprapubic area, skin Pfannenstiel transverse incision, dissection of the anterior rectus sheath transversely and opening the parietal peritoneum longitudinally. Starting adhesionaccords the site of adhesion using scissor dissection and ligation or coagulation diathermy. The choice between coagulation and ligation depend upon characters of adhesions as coagulation was used in the presence of visible thin and a vascular adhesion but ligation was used in presence of incomplete visible band, thick and vascular one. Adhesion can be completed after delivery of the fetus to avoid delay in delivery of the fetus and the remaining adhesions were dissected after exteriorization of the uterus which allowed easy dissection. After the closure of the uterus in 2 layers and good homeostasis, leaving the visceral peritoneum without closure but with meticulous coagulation of its edge, closure of the parietal peritoneum and closure of anterior rectus sheath was done. Two grams of cefotaxime as prophylactic dose before cord cutting were given to every patient. The following data were recorded intra-operatively for all patients; duration of CS, occurrence of morbidity, amount of blood loss, need for blood transfusion, types and criteria of adhesions. Maternal morbidity included all those related directly to adhesion like bladder injury or bladder injury or indirectly like delay of management of severe atonic postpartum hemorrhage due to obscuring adhesion. Neonatal condition at delivery, Apgar score and the need for admission to pediatric care unit (PCU) were also recorded. Postoperative follow-up was done with a recording of the time of passing flatus, duration of hospital stay, need for postoperative analgesia and occurrence of any wound infections. The patients starting diet after 6 hours from CS. All fifty cases were followed up till the next CS for assessment of the site and types of formed adhesions in the next cesarean section. Adhesions are defined as the presence of bands between two pelvic organs or between any pelvic organ and the abdominal wall. According to the inclusion criteria of the study pregnant women evermarried of reproductive aged group were included in this study whereas according to the exclusion criteria severely ill patients were excluded from the study.

\section{RESULTS}

In this study in total 60 patients with repeated CS that were classified as follows: 23 had previous two CS, 28 had previous three CS, 8 had previous four CS and 1 had previous five CS. The mean age of study participants was $31.50 \pm 4.25$ years and the mean parity was 2.64 \pm 0.75 . No cases with maternal morbidity occurred during adhesiolysis. The mean blood loss was not significantly increased $(552 \pm 72.40)$ without and need for blood transfusion in all cases. No cases with neonatal morbidity or admission to ICU. The mean Apgar score at 1 minute was $9.11 \pm 0.50$. The intraoperative adhesions found were variable in sites and types. The mean gestational age of the participants was $38.80 \pm 0.24$ weeks. On the other hand, the mean (with SD) duration of CS and the mean (with SD) hospital staying period were $56.75 \pm 8.25 \mathrm{~min}$. and $8.20 \pm 0.20$ days respectively. The most common type of adhesions was uterovesical found in all cases (100\%). Omental adhesions with the anterior uterine wall came next in 56 cases $(93.33 \%)$. Case of uterosigmoid was $18.33 \%$ and the case with the uterus intestinal was $11.67 \%$ in this study. Besides all these findings in analyzing the sites of adhesions we found the highest $70 \%$ cases of avascular. Then both fine and thin were $38.33 \%$ separately. On the other hand the frequencies of dense, thick, vascular and distort anatomy were $58.33 \%$, $45 \%, 28.33 \%$ and $21.67 \%$ respectively. 


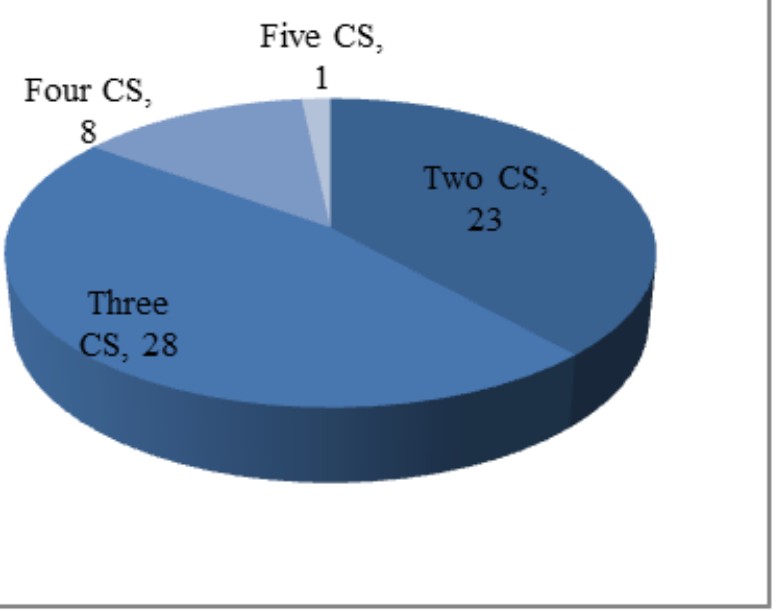

Fig-1: History of previous CS of the participants $(\mathrm{N}=60)$

Table-1: Socio-demographic and clinical features of participants $(\mathrm{N}=60)$

\begin{tabular}{|l|l|}
\hline Factors & Mean \pm SD \\
\hline Age (years) & $31.50 \pm 4.25$ \\
\hline Parity & $2.64 \pm 0.75$ \\
\hline Gestational age (weeks) & $38.80 \pm 0.24$ \\
\hline Duration of CS (min) & $56.75 \pm 8.25$ \\
\hline Amount of blood loss (ml) & $552 \pm 72.40$ \\
\hline Passage of flatus (days) & $1.7 \pm 0.50$ \\
\hline Hospital stay (hours) & $8.20 \pm 0.20$ \\
\hline
\end{tabular}

Table-2: Types of intraoperative adhesions $(\mathrm{N}=60)$

\begin{tabular}{|l|l|l|}
\hline Sites & n & \% \\
\hline Uterovesical & 60 & 100 \\
\hline Omental with the uterus & 56 & 93.33 \\
\hline Uterosigmoid & 11 & 18.33 \\
\hline with the uterus Intestinal & 7 & 11.67 \\
\hline
\end{tabular}

Table-3: Sites of intraoperative adhesions $(\mathrm{N}=60)$

\begin{tabular}{|l|l|l|}
\hline Types & n & \% \\
\hline Fine & 23 & 38.33 \\
\hline Dense & 35 & 58.33 \\
\hline Thin & 23 & 38.33 \\
\hline Thick & 27 & 45 \\
\hline Avascular & 42 & 70 \\
\hline Vascular & 17 & 28.33 \\
\hline Distort anatomy & 13 & 21.67 \\
\hline
\end{tabular}

\section{DISCUSSION}

In our study in total 60 patients with repeated CS that were classified as follows: 23 had previous two CS, 28 had previous three CS, 8 had previous four CS and 1 had previous five CS. The mean age of study participants was $31.50 \pm 4.25$ years and the mean parity was 2.64 \pm 0.75 . No cases with maternal morbidity occurred during adhesiolysis. The mean blood loss was not significantly increased $(552 \pm 72.40)$ without and need for blood transfusion in all cases. No cases with neonatal morbidity or admission to ICU. Clinical data on the efficacy of adhesion prevention strategies related to the setting of CS are limited; also, there is a significant need for well-controlled, randomized clinical studies investigating adhesion prevention in the labor and delivery setting [9]. Gedikbasi et al., 2010 found that there was an increase in the risks for operative complications and poor perinatal outcomes in multiple repeat CS. Patients must be informed about the related risks of multiple repeated CSs and tubal ligation needs to be encouraged [10]. In our study no recorded cases of bladder or intestinal injury related to adhesiolysis, no cases of delayed management of any obstetrics complication related to adhesiolysis. Adhesion before delivery increase the blood loss and need for transfusion during CS was debatable [1]. Although some have suggested that significant blood loss is associated with higher number CSs [11-13]. Other studies were not associated with significant blood loss [14]. While some have found that the risk of blood transfusion increased significantly with increase in the number of prior CSs $[11,12]$ others have either found no difference overall $[12,13]$. In our study, no significant blood loss was recorded in all cases and no need for a blood transfusion at all. Sikirica et al., 2012 found that adhesion rates were higher in repeat compared with primary CS. In repeat CS patients, costs and complications were higher in the adhesion group. Reducing adhesion formation after primary CS could reduce cost and complications at the time of repeat CS [15]. Regarding perinatal morbidity, some studies reported that the mean incision to delivery time in women with high score adhesions was significantly higher, compared to those with low score adhesions (19.8 minutes vs 15.6 minutes, respectively; $\mathrm{p}=0.04)$. Also in the same study analysis, it was found by 30 minutes after skin incision, $17.9 \%$ of women with high score adhesions remained undelivered, versus $5.1 \%$ of those with low score adhesions $(p=0.04)$. Delivery times have also been reported to increase with increase in the number of previous CSs in the same study [16]. All the previous studies found that adhesion increase of maternal injuries during adhesion and also delay interference in case of any complications like doing subtotal hysterectomy instead of total hysterectomy as adhesion distort 
anatomy. In the current study we found the most common type of adhesions was uterovesical found in all cases $(100 \%)$. Omental adhesions with the anterior uterine wall came next in 56 cases $(93.33 \%)$. Case of uterosigmoid was $18.33 \%$ and the case with the uterus intestinal was $11.67 \%$ in this study. Besides all these findings in analyzing the sites of adhesions we found the highest $70 \%$ cases of avascular. Then both fine and thin were $38.33 \%$ separately. On the other hand the frequencies of dense, thick, vascular and distort anatomy were $58.33 \%, 45 \%, 28.33 \%$ and $21.67 \%$ respectively. Operative time was not prolonged with gaining experience of perfect adhesiolysis; also, blood loss was within normal range and no maternal morbidity nor mortality at all. Also, one of the advantages is early discharge within hours without complications. Although there are many factors that have an influence on the postoperative adhesion formation and doing adhesion might not be the factor on recurrent adhesions, we tried to choose a homogenous group of patients with the same pattern of postoperative follow-up to minimize other factors affecting adhesion formation. The limitations of the current study include an absence of a control group, but currently, we are working in a large case-control study based on our preliminary results to confirm our hypothesis. Second, the small sample size was taken that makes our result cannot be generalizable to the population. The most important point gained is the very low incidence of adhesion in next CS and the operation was simple and easy in comparison to past one. The aim of this study was to evaluate the adhesion in previous cesarean sections regarding the sites and types of adhesion. All the intervensions were performed to achieve the goal of this study.

\section{LIMITATIONS OF THE STUDY}

This was a single centered study with a small sized of sample. So the findings of this may not reflect the exact scenario of the whole country.

\section{CONCLUSION AND \\ RECOMMENDATIONS}

A high percentage of cesarean deliveries result in adhesive disease, which delays repeat cesarean delivery of the fetus. The potential for adhesive disease should be included in counseling regarding primary elective cesarean births. The findings may be helpful in the treatment arena and in farther studies. For getting more specific findings we would like to recommend for conducting more studies regarding the same issue with larger sized sample.

\section{REFERENCES}

1. World Health Organization (WHO) (1948) Health for all.

2. Bangladesh Demographic and Health Survey (BDHS). National Institute of Population. 2011.

3. Dietz HP, Bennett MJ. The effect of childbirth on pelvic organ mobility. Obstet Gynecol, 2003; 102:223-8.

4. Hemminki E. Long term maternal health effects of caesarean section. J Epidemiol Comm Health 1991; 45:24-8.

5. Hall MH, Bewley S. Maternal mortality and mode of delivery. The Lancet. 1999 Aug 28;354(9180):776.

6. Hauwa SA. Utilization of maternal health care services in Nigeria: an analysis of regional differences in the patterns and determinants of maternal health care use. 2011.

7. Awusi VO, Anyanwu EB, Okeleke V. Determinants of antenatal care services utilization in emevor village, Nigeria. Benin Journal of Postgraduate Medicine, 2009; 11.

8. Sharma SK, Sawangdee Y, Sirirassamee B. 'Access to health: women's status and utilization of maternal health services in Nepal', Journal of Biosocial Science, Cambridge, 2007: 39:671-692.

9. Sari K. Socio-economic and demographic determinants of maternal health care utilization in Indonesia. 2009.

10. David S. The use of antenatal and postnatal care: perspectives and experiences of women and health care providers in rural southern Tanzania. BMC Pregnancy and Childbirth. 2009; 9:10. http://www.biomedcentral.com/1471-2393/9/10.

11. Worku AG, Yalew AW, Afework MF. Factors affecting utilization of skilled maternal care in Northwest Ethiopia: a multilevel analysis. BMC International Health and Human Rights, 2013; 13:20. http://www.biomedcentral.com/1472$98 \mathrm{X} / 13 / 20$

12. Safe Motherhood Initiative (2003) viewed 28 October 2008, http://www.safemotherhood.org

13. Simon MC, Anwar I, Ronsmans C. A decade of inequality in maternity care. International Journal for Equity in Health. 1991-2004; 6.

14. Determinants of antenatal and delivery care utilization in Tigray region, Ethiopia: a crosssectional study. International Journal for Equity in Health, $12: 30$

http://www.equityhealthj.com/content/12/1/30

15. Birmeta B, Dibaba Y, Woldeyohannes D. Determinants of maternal health care utilization in Holeta town, central Ethiopia. BMC Health Services Research. 2013; 13:256. http://www.biomedcentral.com/1472-6963/13/256

16. International Journal for Equity in Health 6:9http://www.equityhealthj.com/content/6/1/9. 